\title{
Switching of ultrashort pulses in nonlinear high-birefringence two-core optical fibers
}

\author{
Jin Hua Li ${ }^{\mathrm{a} \#}$, Kin Seng Chiang ${ }^{\mathrm{a} *}$, Kwok Wing Chow ${ }^{\mathrm{b} \dagger}$ \\ ${ }^{a}$ CityUHK-UESTC Joint Research Center on Optical Fiber Sensing and Communication and \\ Department of Electronic Engineering, City University of Hong Kong, Hong Kong, China \\ ${ }^{\mathrm{b}}$ Department of Mechanical Engineering, University of Hong Kong, Hong Kong, China \\ *Corresponding author. Email: eeksc@cityu.edu.hk; Tel: +852 34429605 Fax: +852 34420562 \\ \# Email: marylijinhua@gmail.com \\ ${ }^{\dagger}$ Email: kwchow@hku.hk
}

\begin{abstract}
We analyze the switching characteristics of ultrashort pulses in a nonlinear high-birefringence two-core optical fiber by solving a set of four generalized coupled nonlinear Schrödinger equations. In such a fiber, the critical power required for activating switching changes significantly with the polarization angle of the input pulse and, as a result, a pulse at a proper power level can be switched between the two cores of the fiber by changing the input polarization angle. This provides a simple mechanism of achieving optical switching with the fiber. We also study the effects of the group-delay difference (GDD) between the two polarization components and the coupling-coefficient dispersion (CCD) in the fiber on the switching characteristics. The GDD tends to break up the two polarization components in the input pulse and thus leads to an increase in the switching
\end{abstract}


power. A larger GDD, however, can give a sharper switching contrast when the input polarization angle is varied. The CCD tends to break up the input pulse and cause pulse distortion, regardless of the polarization, so it also leads to an increase in the switching power. Unlike the GDD, a large CCD always reduces the switching contrast. To achieve high-quality switching, the fiber should have a small CCD.

Key Words: birefringence, nonlinear pulse propagation, optical switching, polarization, two-core optical fiber 


\section{Introduction}

Nonlinear two-core optical fibers (TCFs) have drawn tremendous attention since the

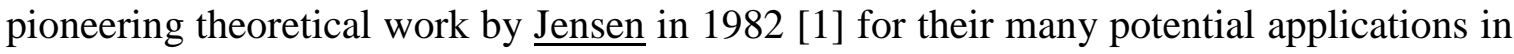
optical signal processing, especially as all-optical switches [2 - 12]. All-optical switching has been experimentally demonstrated in conventional TCFs [2 - 5] and, more recently, in a two-core photonic crystal fiber [6] using high-power ultrashort pulse lasers. To avoid pulse distortion caused by the group velocity dispersion (GVD) in the fiber, the use of soliton pulses for optical switching has been proposed [7]. Many other effects on the switching dynamics in TCFs, such as intermodal dispersion [13, 14], third-order dispersion [15], and intrapulse Raman scattering [16, 17] have been investigated.

A TCF that consists of two identical cores possesses two symmetry axes, and hence the fiber must possess geometry-induced birefringence, which means that the light waves polarized along the two symmetry axes of the fiber propagate at slightly different phase velocities. In addition, because the materials for the cores and the cladding of the fiber have different thermal expansion coefficients, stress-induced birefringence in the fiber is normally produced when the fiber is drawn from the preform at a high temperature. The total birefringence in a TCF is the combined result of the geometry birefringence and the stress birefringence. It is a complicated exercise to analyze a nonlinear birefringent TCF, because four coupled nonlinear equations need to be solved. The majority of the studies on TCFs deal with a simpler problem where the birefringence in the fiber is ignored [1 - 
17]. A zero-birefringence TCF is described by two coupled equations and much more amenable to analytical treatment. There are only a few theoretical studies on birefringent TCFs [18 - 22], which include an analysis of all-optical continuous-wave (CW) switching [18], a study of soliton states [19] and their stability [20] in the absence of group-delay difference (GDD) between the two polarization components, a numerical investigation of vector soliton pulse switching [21], and a detailed analysis of the modulation instabilities in linearly and circularly birefringent TCFs [22]. In spite of these studies, the role of the birefringence in a TCF in affecting the switching characteristics of ultrashort pulses is yet to be fully understood.

The primary objective of this paper is to study the switching characteristics of a nonlinear high-birefringence (Hi-Bi) TCF with an emphasis on the understanding of the effects of the polarization state of the input pulse. Our analysis is based on numerically solving a set of four generalized coupled nonlinear Schrödinger equations which incorporate the effects of the GDD and the coupling-coefficient dispersion (CCD) in the fiber. The significance of these effects on the switching performance of ultrashort pulses is investigated. Our main results are summarized below:

- The critical power required for activating switching changes significantly with the polarization angle. This makes possible the realization of polarization-activated switching, where a pulse at an appropriate power level is switched between the two cores by varying the polarization angle.

- The GDD increases the switching power and is undesirable for power-controlled 
switching. However, it increases the switching contrast for polarization-activated switching.

- The CCD increases the switching power and reduces the switching contrast, and should be minimized for both power-controlled and polarization-activated switching.

Modern TCFs based on photonic crystal structures can be designed to possess strong birefringence by adjusting the arrangement and the size of the air holes around the core region [23]. A study of all-optical switching in such fibers must take into account the effects of the birefringence. In fact, the recent experimental demonstration of all-optical switching in a 9-mm long two-core photonic crystal fiber using 120-fs laser pulses (at a wavelength around $1550 \mathrm{~nm}$ with peak intensity up to a few TW/ $\mathrm{cm}^{2}$ ) exhibits significant polarization dependence [6]. With such ultrashort pulses, the effects of the GDD and the CCD in the fiber, both of which tend to break up the pulses, are expected to be important. The findings in this paper can provide insight into the switching behavior of a $\underline{\mathrm{Hi}-\mathrm{Bi}} \mathrm{TCF}$ and thus facilitate the design of fibers and experiments to demonstrate the polarization-activated switching effects.

\section{Coupled-mode equations}

We consider a lossless TCF with two identical single-mode cores aligned in the $x$ direction, where the light waves in the fiber propagate in the $z$ direction. Because of the presence of the birefringence in the fiber, there are two orthogonal polarization modes in each core, the $x$-polarization mode and the $y$-polarization mode, which propagate along 
the fiber at different phase velocities. A pulse with an arbitrary polarization state launched into one of the cores can be decomposed into the two principal polarization components. The propagation of the pulse in such a fiber is described by the following coupled nonlinear Schrödinger equations, which can be obtained by extending the existing equations for TCFs [7 - 11]:

$$
\begin{aligned}
& i\left(\frac{\partial a_{1 x}}{\partial z}+\beta_{1 x} \frac{\partial a_{1 x}}{\partial t}\right)-\frac{1}{2} \beta_{2 x} \frac{\partial^{2} a_{1 x}}{\partial t^{2}}+\gamma_{x}\left(\left|a_{1 x}\right|^{2}+\frac{2}{3}\left|a_{1 y}\right|^{2}\right) a_{1 x} \\
& +\frac{\gamma_{x}}{3} a_{1 y}^{2} a_{1 x}^{*} \exp \left[-2 i\left(\beta_{0 x}-\beta_{0 y}\right) z\right]+C_{x} a_{2 x}+i C_{1 x} \frac{\partial a_{2 x}}{\partial t}=0, \\
& i\left(\frac{\partial a_{2 x}}{\partial z}+\beta_{1 x} \frac{\partial a_{2 x}}{\partial t}\right)-\frac{1}{2} \beta_{2 x} \frac{\partial^{2} a_{2 x}}{\partial t^{2}}+\gamma_{x}\left(\left|a_{2 x}\right|^{2}+\frac{2}{3}\left|a_{2 y}\right|^{2}\right) a_{2 x} \\
& +\frac{\gamma_{x}}{3} a_{2 y}^{2} a_{2 x}^{*} \exp \left[-2 i\left(\beta_{0 x}-\beta_{0 y}\right) z\right]+C_{x} a_{1 x}+i C_{1 x} \frac{\partial a_{1 x}}{\partial t}=0, \\
& i\left(\frac{\partial a_{1 y}}{\partial z}+\beta_{1 y} \frac{\partial a_{1 y}}{\partial t}\right)-\frac{1}{2} \beta_{2 y} \frac{\partial^{2} a_{1 y}}{\partial t^{2}}+\gamma_{y}\left(\left|a_{1 y}\right|^{2}+\frac{2}{3}\left|a_{1 x}\right|^{2}\right) a_{1 y} \\
& +\frac{\gamma_{y}}{3} a_{1 x}^{2} a_{1 y}^{*} \exp \left[2 i\left(\beta_{0 x}-\beta_{0 y}\right) z\right]+C_{y} a_{2 y}+i C_{1 y} \frac{\partial a_{2 y}}{\partial t}=0 \\
& i\left(\frac{\partial a_{2 y}}{\partial z}+\beta_{1 y} \frac{\partial a_{2 y}}{\partial t}\right)-\frac{1}{2} \beta_{2 y} \frac{\partial^{2} a_{2 y}}{\partial t^{2}}+\gamma_{y}\left(\left|a_{2 y}\right|^{2}+\frac{2}{3}\left|a_{2 x}\right|^{2}\right) a_{2 y} \\
& +\frac{\gamma_{y}}{3} a_{2 x}^{2} a_{2 y}^{*} \exp \left[2 i\left(\beta_{0 x}-\beta_{0 y}\right) z\right]+C_{y} a_{1 y}+i C_{1 y} \frac{\partial a_{1 y}}{\partial t}=0 .
\end{aligned}
$$

In the above equations, $a_{j p}(j=1,2$ and $p=x, y)$, a function of $z$ and $t$, is the slowly varying amplitude of the electric field of the $p$-polarization mode in the $j$-th core; $z$ and $t$ 
are the distance and time coordinates, respectively; $\beta_{0 p}$ is the propagation constant of the $p$-polarization mode; $\beta_{1 p}$ is the group delay of the $p$-polarization mode; $\beta_{2 p}$ is the GVD with $\beta_{2 p}<0$ and $\beta_{2 p}>0$ for anomalous and normal dispersion, respectively; $\gamma_{p}$ is the nonlinear coefficient of the $p$ polarization; $C_{p}$ is the linear coupling coefficient of the $p$ polarization, which is responsible for the periodic power transfer between the two cores in a linear TCF; $C_{1 p}=\mathrm{d} C_{p} / \mathrm{d} \omega$, evaluated at the optical carrier frequency of the pulse, represents the coupling-coefficient dispersion (CCD), which is equivalent to the intermodal dispersion arising from the group delay difference in the $p$ polarization between the even and odd supermodes of the TCF $[24,25]$. The first and second nonlinear terms in each of the above equations account for the self-phase modulation (SPM) and cross-phase modulation (XPM) effects, respectively. The third nonlinear term comes from the nonlinear coherent coupling between the two orthogonal polarization components in each core.

In the case that the birefringence in the fiber, namely $\left|\beta_{0 x}-\beta_{0 y}\right|$, is large, the nonlinear coherent coupling term oscillates rapidly along the propagation direction and its contribution is averaged out to zero [18], which suggests that this term can be ignored for a Hi-Bi TCF. In practice, the polarization dependences of the GVD, the nonlinearity coefficient, and the coupling coefficient dispersion, are weak, so we can put $\beta_{2 x}=\beta_{2 y} \equiv \beta_{2}$, $\gamma_{x}=\gamma_{y} \equiv \gamma$, and $C_{1 x}=C_{1 y} \equiv C_{1}$. With these assumptions, Eq. (1) is simplified to the following set of equations: 


$$
\begin{aligned}
& i\left(\frac{\partial a_{1 x}}{\partial z}+\beta_{1 x} \frac{\partial a_{1 x}}{\partial t}\right)-\frac{1}{2} \beta_{2} \frac{\partial^{2} a_{1 x}}{\partial t^{2}}+\gamma\left(\left|a_{1 x}\right|^{2}+\frac{2}{3}\left|a_{1 y}\right|^{2}\right) a_{1 x}+C a_{2 x}+i C_{1} \frac{\partial a_{2 x}}{\partial t}=0 \\
& i\left(\frac{\partial a_{2 x}}{\partial z}+\beta_{1 x} \frac{\partial a_{2 x}}{\partial t}\right)-\frac{1}{2} \beta_{2} \frac{\partial^{2} a_{2 x}}{\partial t^{2}}+\gamma\left(\left|a_{2 x}\right|^{2}+\frac{2}{3}\left|a_{2 y}\right|^{2}\right) a_{2 x}+C a_{1 x}+i C_{1} \frac{\partial a_{1 x}}{\partial t}=0 \\
& i\left(\frac{\partial a_{1 y}}{\partial z}+\beta_{1 y} \frac{\partial a_{1 y}}{\partial t}\right)-\frac{1}{2} \beta_{2} \frac{\partial^{2} a_{1 y}}{\partial t^{2}}+\gamma\left(\left|a_{1 y}\right|^{2}+\frac{2}{3}\left|a_{1 x}\right|^{2}\right) a_{1 y}+C a_{2 y}+i C_{1} \frac{\partial a_{2 y}}{\partial t}=0 \\
& i\left(\frac{\partial a_{2 y}}{\partial z}+\beta_{1 y} \frac{\partial a_{2 y}}{\partial t}\right)-\frac{1}{2} \beta_{2} \frac{\partial^{2} a_{2 y}}{\partial t^{2}}+\gamma\left(\left|a_{2 y}\right|^{2}+\frac{2}{3}\left|a_{2 x}\right|^{2}\right) a_{2 y}+C a_{1 y}+i C_{1} \frac{\partial a_{1 y}}{\partial t}=0
\end{aligned}
$$

By introducing the normalized parameters:

$$
Z=\frac{Z}{L_{D}}, \quad T=\frac{1}{T_{0}}\left[t-\left(\frac{\beta_{1 x}+\beta_{1 y}}{2}\right) Z\right], \quad A_{j p}=\sqrt{\gamma L_{D}} \cdot a_{j p}, \quad L_{D}=\frac{T_{0}^{2}}{\left|\beta_{2}\right|},
$$

where $T_{0}$ is a characteristic width of the input pulse and $L_{D}$ is the dispersion length, Eq.

(2), assuming anomalous GVD $\left(\beta_{2}<0\right)$, can be expressed in the normalized form:

$$
\begin{aligned}
& i\left(\frac{\partial A_{1 x}}{\partial Z}+\delta \frac{\partial A_{1 x}}{\partial T}\right)+\frac{1}{2} \frac{\partial^{2} A_{1 x}}{\partial T^{2}}+\left(\left|A_{1 x}\right|^{2}+\frac{2}{3}\left|A_{1 y}\right|^{2}\right) A_{1 x}+R A_{2 x}+i R_{1} \frac{\partial A_{2 x}}{\partial T}=0, \\
& i\left(\frac{\partial A_{2 x}}{\partial Z}+\delta \frac{\partial A_{2 x}}{\partial T}\right)+\frac{1}{2} \frac{\partial^{2} A_{2 x}}{\partial T^{2}}+\left(\left|A_{2 x}\right|^{2}+\frac{2}{3}\left|A_{2 y}\right|^{2}\right) A_{2 x}+R A_{1 x}+i R_{1} \frac{\partial A_{1 x}}{\partial T}=0, \\
& i\left(\frac{\partial A_{1 y}}{\partial Z}-\delta \frac{\partial A_{1 y}}{\partial T}\right)+\frac{1}{2} \frac{\partial^{2} A_{1 y}}{\partial T^{2}}+\left(\left|A_{1 y}\right|^{2}+\frac{2}{3}\left|A_{1 x}\right|^{2}\right) A_{1 y}+R A_{2 y}+i R_{1} \frac{\partial A_{2 y}}{\partial T}=0, \\
& i\left(\frac{\partial A_{2 y}}{\partial Z}-\delta \frac{\partial A_{2 y}}{\partial T}\right)+\frac{1}{2} \frac{\partial^{2} A_{2 y}}{\partial T^{2}}+\left(\left|A_{2 y}\right|^{2}+\frac{2}{3}\left|A_{2 x}\right|^{2}\right) A_{2 y}+R A_{1 y}+i R_{1} \frac{\partial A_{1 y}}{\partial T}=0,
\end{aligned}
$$

with 


$$
\begin{aligned}
& \delta=\frac{\beta_{1 x}-\beta_{1 y}}{2\left|\beta_{2}\right|} T_{0}=\operatorname{sgn}\left(\beta_{1 x}-\beta_{1 y}\right) \frac{L_{D}}{2 L_{W G}}, \\
& R=\frac{C}{\left|\beta_{2}\right|} T_{0}^{2}=\frac{\pi}{2} \frac{L_{D}}{L_{C}},
\end{aligned}
$$

and

$$
R_{1}=\frac{C_{1}}{\left|\beta_{2}\right|} T_{0}=\operatorname{sgn}\left(C_{1}\right) \frac{L_{D}}{2 L_{W C}},
$$

where the coupling length $L_{C}$ and the walk-off lengths caused by the GDD and the CCD, $\underline{L}_{W G}$ and $L_{W C}$, are defined, respectively, by

$$
\begin{aligned}
& L_{C}=\frac{\pi}{2 C}, \\
& L_{W G}=\frac{T_{0}}{\left|\beta_{1 x}-\beta_{1 y}\right|}, \\
& L_{W C}=\frac{T_{0}}{2\left|C_{1}\right|} .
\end{aligned}
$$

The normalized amplitudes $A_{j p}(j=1,2$ and $p=x, y)$ in Eq. (3) are functions of the normalized distance and retarded time coordinates, $Z$ and $T$. The parameter $\delta$, defined by Eq. (4), is a measure of the GDD between the two orthogonal polarization components in each core. Without loss of generality, we assume $\delta \geq 0$. It is possible to design a Hi-Bi fiber with $\delta$ over a wide range (including a zero value) by properly controlling the geometry birefringence and the stress birefringence in the fiber [26]. The parameters $R$ and $R_{1}$, defined by Eq. (5) and Eq. (6), respectively, are the normalized linear coupling 
coefficient and CCD, respectively.

The normalization procedure can reduce the number of free parameters and thus allows a more general discussion of the switching behavior. As shown by Eqs. (4) - (6), the GDD, the linear coupling coefficient, and the CCD are all normalized with respect to the GVD scaled with the pulse width, which effectively leads to a comparison of the characteristic lengths. In the case $R>>R_{1}$ and $\delta$, i.e., $L_{\underline{C}}<<L_{W C} \underline{\text { and }} \underline{L}_{W G} \underline{\text { which }}$ corresponds to the use of long pulses ( $>>100 \mathrm{fs}$ ) and/or strongly coupled cores, it takes a large number of coupling lengths to observe significant pulse breakup caused by the GDD and the CCD and, as a result, the GDD and the CCD have little effects on optical switching with a short fiber (less than a few coupling lengths). In our study, we take $R=1$ and $-1 \leq R_{1} \leq 0$, which corresponds to the practical situation of launching a $\sim 100$-fs pulse at the wavelength $1.5 \mu \mathrm{m}$ into a typical TCF with $\beta_{2}=20 \mathrm{ps}^{2} / \mathrm{km}, \gamma=3 /(\mathrm{W} \cdot \mathrm{km})$, and a center-to-center core separation of approximately 6 to 8 times of the core radius [11]. The range of the GDD values should be similar to that in a single-core $\mathrm{Hi}-\mathrm{Bi}$ fiber and, consequently, is taken as $0 \leq \delta \leq 2.5$ [27].

We solve Eq. (3) numerically with the pseudospectral method in the time domain and the fourth-order Runge-Kutta scheme with an adaptive step-size control in the space domain [28].

\section{Results and discussions}

We consider a pulse launched into only one core of a Hi-Bi TCF: 


$$
\begin{aligned}
& A_{1 x}(0, T)=\sqrt{P_{0}} \cos (\theta) \cdot \operatorname{sech}(T), \\
& A_{1 y}(0, T)=\sqrt{P_{0}} \sin (\theta) \cdot \sec h(T), \\
& A_{2 x}(0, T)=0 \text {, } \\
& A_{2 y}(0, T)=0 \text {, }
\end{aligned}
$$

where $P_{0}$ is the normalized peak power of the input pulse $\left(P_{0}=1\right.$ is known as the soliton power) and $\theta$ is the polarization angle that determines the relative strength of the two polarization components in the input pulse. We calculate the transmission coefficient of the fiber for each core, which is defined as the output energy from that core at a propagation distance of one coupling length divided by the total energy of the input pulse, namely,

$$
T_{j}=\frac{\int_{-\infty}^{+\infty}\left(\left|A_{j x}\left(L_{R}, T\right)\right|^{2}+\left|A_{j y}\left(L_{R}, T\right)\right|^{2}\right) d T}{\int_{-\infty}^{+\infty}\left(\left|A_{1 x}(0, T)\right|^{2}+\left|A_{1 y}(0, T)\right|^{2}\right) d T}, \quad j=1,2
$$

where $\underline{L}_{R}=\pi /(2 R)$ is the normalized coupling length.

\subsection{Effects of the polarization angle}

In this section, we study the effects of the polarization angle $\theta$ on the switching characteristics of a Hi-Bi TCF, where the CCD is assumed to be zero, i.e., $R_{1}=0$.

We first consider the case where the input pulse contains only one principal polarization component, i.e., $\theta=0^{\circ}$ or $90^{\circ}$. Figure 1 shows the dependence of the 
transmissions of the fiber on the input pulse power for this case with $R=1$ and $R_{1}=0$. The results shown in Fig. 1 are independent of the value of $\delta$ and identical to the published results for a zero-birefringence TCF $[8,11]$. This is expected, because, for the single principal polarization input, the birefringence and the GDD have no effects on the pulse propagation; the four equations given in Eq. (4) are reduced to a set of two coupled equations, the same equations that govern pulse propagation in a zero-birefringence TCF. These results verify the validity of our computer program and, at the same time, serve as the reference for subsequent discussions. In the linear limit ( $P_{0}$ approaches zero), the input pulse is transferred completely from the input core to the cross core, i.e., $T_{1}=0$ and $T_{2}=1$, as expected for a one-coupling-length TCF. As the input power increases over a critical value, much of the input pulse is switched from the cross core to the input core. As shown in Fig. 1, the switching power $P_{\mathrm{sw}}$, defined as the normalized input power required for the transmitted powers from the two cores to be equal, i.e., $T_{1}=T_{2}=0.5$, is equal to 3.61.

We next consider the cases where the input pulse contains both principal polarization components, i.e., $0^{\circ}<\theta<90^{\circ}$. The results for $\theta$ and $\left(90^{\circ}-\theta\right)$ are identical by symmetry. In these cases, the pulse propagation dynamics is affected by the GDD in the fiber. Figures 2(a) - (f) show six sets of switching characteristics of a Hi-Bi TCF at $\theta=15^{\circ}$ $\left(75^{\circ}\right), 30^{\circ}\left(60^{\circ}\right)$, and $45^{\circ}$, which correspond to $\delta=0,0.5,1.0,1.5,2.0$, and 2.5, respectively. At any given value of $\delta$, the switching power increases, as the polarization angle $\theta$ increases from $0^{\circ}$ to $45^{\circ}$ (or decreases from $90^{\circ}$ to $45^{\circ}$ ) with the value at $\theta=45^{\circ}$ 
being the largest. For example, for a Hi-Bi TCF without $\underline{\text { GDD, }}$, i.e., $\delta=0$, we have $P_{\mathrm{sw}}=$ 3.61 (Fig. 1), 3.74, 4.09, and 4.33 for $\theta=0^{\circ}\left(90^{\circ}\right), 15^{\circ}\left(75^{\circ}\right), 30^{\circ}\left(60^{\circ}\right)$, and $45^{\circ}$. We can understand this phenomenon intuitively. When the power of the input pulse is distributed between the two polarizations, the GDD broadens the pulse along the fiber and thus weakens the nonlinear effect. A higher input power is therefore needed to counteract the GDD effect to activate switching. As the GDD effect is strongest at $\theta=45^{\circ}$, the critical switching power is highest at $\theta=45^{\circ}$. The effects of the GDD are discussed in detail in the next section.

\subsection{Effects of the group-delay difference (GDD)}

The effects of the GDD between the two polarization components are shown in Fig. 2(a) - (f). By comparing the switching characteristics at the same polarization angle for different values of $\delta$ in Fig. 2, we find that the switching power at a given polarization angle (except at $0^{\circ}$ or $90^{\circ}$ ) increases with the value of $\delta$. The increase becomes more and more significant as the polarization angle approaches $45^{\circ}$. The results are summarized in Fig. 3, where the switching power is plotted as a function of $\delta$. This increase of the switching power with $\delta$ can be explained by the tendency of the GDD to break up the two principal polarization components in the input pulse. Consequently, a larger GDD will imply a higher power to "glue" the two polarization components together and activate switching. Our results are consistent with the previous observation that the walk-off effect caused by the GDD in a single-core Hi-Bi fiber can be suppressed by nonlinearity, i.e., by using a sufficiently high input power [29]. We also notice that, when the GDD becomes 
very large $\left(\delta \geq 1.0\right.$ ), the change in the switching characteristics at around $45^{\circ}$ becomes particularly pronounced. When $\theta$ is not close to $45^{\circ}$, the switching contrast, i.e., the maximum difference between $T_{1}$ and $T_{2}$ at a power level exceeding $P_{\mathrm{sw}}$, decreases with an increase in the value of $\delta$, as shown by the first (for $\theta=15^{\circ}$ ) and second (for $\theta=30^{\circ}$ ) columns of the results in Fig. 2. When $\theta$ gets close to $45^{\circ}$, however, the switching contrast becomes large when $\delta \geq 1.0$, as shown by the third column of the results (for $\theta=45^{\circ}$ ) in Fig. 2.

As the switching power is sensitive to the polarization angle, it is possible to achieve switching between the two cores by controlling the polarization angle. For such polarization-activated switching to be effective, the input power must be set at a proper value. Figure 4 shows the variations of the transmissions $T_{1}$ and $T_{2}$ with the polarization angle $\theta$ at different values of $\delta$, where the input power is set at $P_{0}=3.99$, which is somewhat higher than the switching power 3.61 at $\theta=0^{\circ}\left(90^{\circ}\right)$. As shown in Fig. 4, a larger GDD gives a higher switching contrast as $\theta$ is varied, which can be explained by the increase in the switching power with the GDD as $\theta$ approaches $45^{\circ}$. Consequently, a larger GDD is more desirable for polarization-activated switching.

\subsection{Effects of the coupling coefficient dispersion (CCD)}

It has been shown that the CCD in a zero-birefringence TCF tends to break up the input pulse and thus deteriorate the switching characteristics of the fiber for ultrashort pulses, as confirmed both theoretically $[10,11]$ and experimentally $[13,14]$. We should expect similar effects in a Hi-Bi TCF. Figures 5(a) - (c) show the switching 
characteristics of a Hi-Bi TCF with $\delta=0.5, R=1$, and $R_{1}=-1$ at $\theta=0^{\circ}, 22.5^{\circ}$, and $45^{\circ}$, respectively. A comparison with Fig. 1 confirms that the CCD indeed deteriorates the switching characteristics; it tends to increase the switching power and reduce the switching contrast, as in the case of a zero-birefringence TCF [11]. The effects are more significant as the polarization angle approaches $45^{\circ}$, as shown in Fig. 5. Fortunately, the CCD in a TCF can be minimized by properly choosing the physical parameters of the fiber (the core separation, the core diameter, the refractive-index difference between the core and the cladding, and the operation wavelength), which should give sufficient flexibility to design a TCF with a minimal CCD [24]. Photonic crystal TCFs can offer even more flexibility in the control of the birefringence and the CCD in the fiber [30].

\subsection{Soliton pulse switching}

The input pulse given by Eq. (10), which is widely used in the study of all-optical

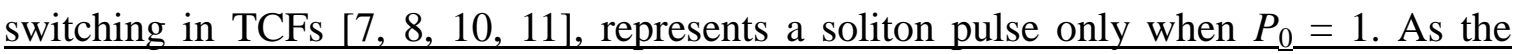
power changes, a soliton pulse should contain a phase that scales simultaneously with the peak power of the pulse. The use of soliton pulses can produce quantitatively better switching characteristics in a zero-birefringence TCF [31]. However, it is not convenient to use soliton pulses to perform all-optical switching experiments, as it is difficult, in practice, to adjust the phase of the input pulse to match its peak power when the pulse power is varied. The switching characteristics presented in our study should not be different qualitatively for soliton pulses. 


\subsection{Alignment of fiber cores}

Our analysis assumes a practical fiber where the two fiber cores are identical in shape with at least two-fold symmetry (e.g., elliptical cores) and the corresponding principal axes of the two cores are aligned. When the principal axes of the two cores are not aligned, the symmetry of the composite structure is broken and the model based on Eq. (1) is no longer valid. In the case where one core is rotated by $90^{\circ}$ from the other core, i.e., the major axis of one core is aligned with the minor axis of the other core, the modes of the same polarization in the individual cores are no longer phase-matched. Each linear coupling term in Eq. (1) includes a phase-mismatch factor determined by the birefringence in each core. The coupled-mode equations for this case (with the CCD $\underline{\text { effects ignored) can be written as }}$

$$
\begin{aligned}
& i\left(\frac{\partial a_{1 x}}{\partial z}+\beta_{1 x} \frac{\partial a_{1 x}}{\partial t}\right)-\frac{\beta_{2}}{2} \frac{\partial^{2} a_{1 x}}{\partial t^{2}}+\gamma\left(\left|a_{1 x}\right|^{2}+\frac{2}{3}\left|a_{1 y}\right|^{2}\right) a_{1 x} \\
& +\frac{\gamma}{3} a_{1 y}{ }^{2} a_{1 x} * \exp \left[-2 i\left(\beta_{0 x}-\beta_{0 y}\right) z\right]+C_{12 x} \exp \left[i\left(\beta_{0 x}-\beta_{0 y}\right) z\right] a_{2 x}=0 \\
& \underline{i\left(\frac{\partial a_{2 x}}{\partial z}+\beta_{1 y} \frac{\partial a_{2 x}}{\partial t}\right)-\frac{\beta_{2}}{2} \frac{\partial^{2} a_{2 x}}{\partial t^{2}}+\gamma\left(\left|a_{2 x}\right|^{2}+\frac{2}{3}\left|a_{2 y}\right|^{2}\right) a_{2 x}} \\
& +\frac{\gamma}{3} a_{2 y}{ }^{2} a_{2 x} * \exp \left[2 i\left(\beta_{0 x}-\beta_{0 y}\right) z\right]+C_{21 x} \exp \left[-i\left(\beta_{0 x}-\beta_{0 y}\right) z\right] a_{1 x}=0 \\
& i\left(\frac{\partial a_{1 y}}{\partial z}+\beta_{1 y} \frac{\partial a_{1 y}}{\partial t}\right)-\frac{\beta_{2}}{2} \frac{\partial^{2} a_{1 y}}{\partial t^{2}}+\gamma\left(\left|a_{1 y}\right|^{2}+\frac{2}{3}\left|a_{1 x}\right|^{2}\right) a_{1 y} \\
& +\frac{\gamma}{3} a_{1 x}{ }^{2} a_{1 y} * \exp \left[2 i\left(\beta_{0 x}-\beta_{0 y}\right) z\right]+C_{12 y} \exp \left[-i\left(\beta_{0 x}-\beta_{0 y}\right) z\right] a_{2 y}=0
\end{aligned}
$$




$$
\begin{aligned}
\frac{i\left(\frac{\partial a_{2 y}}{\partial z}+\beta_{1 x} \frac{\partial a_{2 y}}{\partial t}\right)-\frac{\beta_{2}}{2} \frac{\partial^{2} a_{2 y}}{\partial t^{2}}+\gamma\left(\left|a_{2 y}\right|^{2}+\frac{2}{3}\left|a_{2 x}\right|^{2}\right) a_{2 y}}{+\frac{\gamma}{3} a_{2 x}{ }^{2} a_{2 y} * \exp \left[-2 i\left(\beta_{0 x}-\beta_{0 y}\right) z\right]+C_{21 y} \exp \left[i\left(\beta_{0 x}-\beta_{0 y}\right) z\right] a_{1 y}=0}
\end{aligned}
$$

The four coupling coefficients, $C_{12 x}, C_{12 x}, C_{12 y}$ and $C_{21 y}$ are in general different, but, in practice, we may assume $C_{12 x}=C_{12 y}$ and $C_{21 x} \equiv C_{21 y}$. In the case that the birefringence $\left.\mid \underline{\beta}_{0 x}-\beta_{0 y}\right\rfloor$ is large, the phase-mismatch factors in the linear coupling terms (as well as the nonlinear coherent coupling terms) oscillate rapidly along the propagation direction and, as a result, the linear coupling effect is averaged out to zero, which suggests that the two cores are no longer coupled and effective switching between the two cores is no longer feasible. Consequently, a rotation of one of the cores by $90^{\circ}$ is equivalent to introducing a large dissimilarity between the two cores and, thus, weakens the coupling and switching effects. The analysis of this configuration for weak or moderate birefringence, where the phase-mismatched terms cannot be ignored, is beyond the scope of this paper.

\section{Conclusions}

We have analyzed in detail the switching characteristics of a nonlinear coupling-length Hi-Bi TCF for ultrashort pulses with an emphasis on the understanding of the effects of the polarization angle, the GDD, and the CCD. We show that the switching power increases as the polarization angle increases from $0^{\circ}$ to $45^{\circ}$ (or decreases from $90^{\circ}$ to $45^{\circ}$ ) with a maximum value at $45^{\circ}$. This property of the fiber allows the input pulse set 
at a proper power level to be switched between the two cores by varying only the polarization angle. This mechanism could be explored as a means to achieve optical switching. We find that the switching power increases with the GDD in the fiber, yet a large GDD can increase the transmission contrast between the two cores as the polarization angle is varied. We also show that a large CCD in the fiber tends to reduce the switching contrast and thus deteriorate the switching characteristics. In terms of application, when the fiber is used as a power-dependent switch, the GDD should be minimized. When the fiber is used as a polarization-activated switch, however, a large GDD is preferred. In either case, we should use a fiber that has a smallest possible CCD [24, 31]. We should emphasize that effective switching can be achieved with a coupling length of the TCF, which is translated into a physical length of only several millimeters to several tens of centimeters for typical TCF designs. A two-core photonic crystal fiber can serve as an ideal platform to demonstrate the nonlinear switching effects discussed in this paper.

\section{Acknowledgement}

Partial financial support has been provided by the Research Grants Council contract HKU711713E and the CityU CFSC Project No. 9360146/9687001. 


\section{References}

[1] S. M. Jensen, IEEE J. Quantum Electron. QE-18 (1982) 1580 - 1583.

[2] D. D. Gusovskiǔ, E. M. Dianov, A. A. Maǐer, V. B. Neustruev, E. I. Shklovskiǐ, and I. A. Shcherbakov, Sov. J. Quantum Electron. 15 (1985) 1523 - 1526.

[3] D. D. Gusovskiı̌, E. M. Dianov, A. A. Maǐer, V. B. Neustruev, V. V. Osiko, A. M. Prokhorov, K. Yu. Sitarskiǐ, and I. A. Shcherbakov, Sov. J. Quantum Electron. 17 (1987) $724-727$.

[4] S. R. Friberg, Y. Silberberg, M. K. Oliver, M. J. Andrejco, M. A. Saifi, and P. W. Smith, Appl. Phys. Lett. 51 (1987) 1135 - 1137.

[5] S. R. Friberg, A. M. Weiner, Y. Silberberg, B. G. Sfez, and P. S. Smith, Opt. Lett. 13 (1988) $904-906$.

[6] A. Betlej, S. Suntsov, K. G. Makris, L. Jankovic, D. N. Christodoulides, and G. I. Stegeman, J. Fini, R. T. Bise, and D. J. DiGiovanni, Opt. Lett. 31 (2006) 1480 1482.

[7] S. Trillo, S. Wabnitz, E. M. Wright, and G. I. Stegeman, Opt. Lett. 13 (1988) 672 674.

[8] J. M. Soto-Crespo and E. M. Wright, J. Appl. Phys. 70 (1991) 7240 - 7243.

[9] N. Akhmediev and J. M. Soto-Crespo, Phys. Rev. E 49 (1994) 4519 - 4529. 
[10] P. Shum, K. S. Chiang, and W. A. Gambling, IEEE J. Quantum Electron. 35 (1999) $79-83$.

[11] M. Liu and K. S. Chiang, IEEE J. Quantum Electron. 47 (2011) 1499 - 1505.

[12] N. J. Doran and D. Wood, J. Opt. Soc. Am. B 4 (1987) 1843 - 1846.

[13] K. S. Chiang, Y. T. Chow, D. J. Richardson, D. Taverner, L. Dong, L. Reekie, and K. M. Lo, Opt. Commun. 143 (1997) 189 - 192.

[14] P. Peterka, P. Honzatko, J. Kanka, V. Matejec, and I. Kasik, Proc. SPIE 5036 (2003) $376-381$.

[15] G. L. Diankov, I. M. Uzunov, and F. Lederer, Electron. Lett. 30 (1994) 155 - 156.

[16] B. A. Umarov, F. Kh. Abdullaev, and M. R. B. Wahiddin, Opt. Commun. 162 (1999) $340-346$.

[17] B. A. Malomed, I. M. Skinner, and R. S. Tasgal, Opt. Commun. 139 (1997) 247 251.

[18] S. Trillo and S. Wabnitz, J. Opt. Soc. Am. B 5 (1988) 483 - 491.

[19] T. I. Lakoba, D. J. Kaup, and B. A. Malomed, Phys. Rev. E 55 (1997) 6107 - 6120.

[20] T. I. Lakoba and D. J. Kaup, Phys. Rev. E 56 (1997) 4791 - 4802.

[21] K. S. Amarendra, Opt. Commn. 284 (2011) 186 - 190. 
[22] J. H. Li, K. S. Chiang, B. A. Malomed, and K. W. Chow, J. Phys. B: At. Mol. Opt. Phys. 45 (2012) 165404.

[23] L. Zhang and C. X. Yang, Opt. Express 11 (2003) 1015 - 1020.

[24] K. S. Chiang, Opt. Lett. 20 (1995) 997 - 999.

[25] K. S. Chiang, J. Opt. Soc. Am. B 14 (1997) 1437 - 1443.

[26] K. S. Chiang, Electron. Lett. 21 (1985) 916 - 917.

[27] S. Wabnitz, Phys. Rev. A 38 (1988) 2018 - 2021.

[28] L. D. Carr, J. N. Kutz, and W. P. Reinhardt, Phys. Rev. E 63 (2001) 066604.

[29] C. R. Menyuk, J. Opt. Soc. Am. B 5 (1988) 392 - 402.

[30] M. Liu and K. S. Chiang, Opt. Commun. 293 (2013) 49 - 53.

[31] P. M. Ramos and C. R. Paiva, J. Opt. Soc. Am. B 17 (2000) 1125 - 1133. 


\section{Figure captions}

Fig. 1. Switching characteristics of a coupling-length Hi-Bi TCF with $R=1$ and $R_{1}=0$ at $\theta=0^{\circ}$ or $90^{\circ}$.

Fig. 2. Switching characteristics of a coupling-length Hi-Bi TCF with $R=1$ and $R_{1}=0$ at $\theta=15^{\circ}\left(75^{\circ}\right), 30^{\circ}\left(60^{\circ}\right)$, and $45^{\circ}$ for (a) $\delta=0$, (b) $\delta=0.5$, (c) $\delta=1.0$, (d) $\delta=1.5$, (e) $\delta=2.0$, and (f) $\delta=2.5$.

Fig. 3. Dependence of the switching power $P_{\mathrm{sw}}$ in a coupling-length Hi-Bi TCF with $R=$ 1 and $R_{1}=0$ on the polarization angle $\theta$ at different values of $\delta$.

Fig. 4. Dependence of the transmissions $T_{1}$ and $T_{2}$ of a coupling-length Hi-Bi TCF with $R$ $=1$ and $R_{1}=0$ on the polarization angle $\theta$ at different values of $\delta$, where the input power is set at $P_{0}=3.99$.

Fig. 5. Switching characteristics of a coupling-length Hi-Bi TCF with $\delta=0.5, R=1$, and

$$
R_{1}=-1 \text { at (a) } \theta=0^{\circ} \text {, (b) } \theta=22.5^{\circ} \text {, and (c) } \theta=45^{\circ} \text {. }
$$




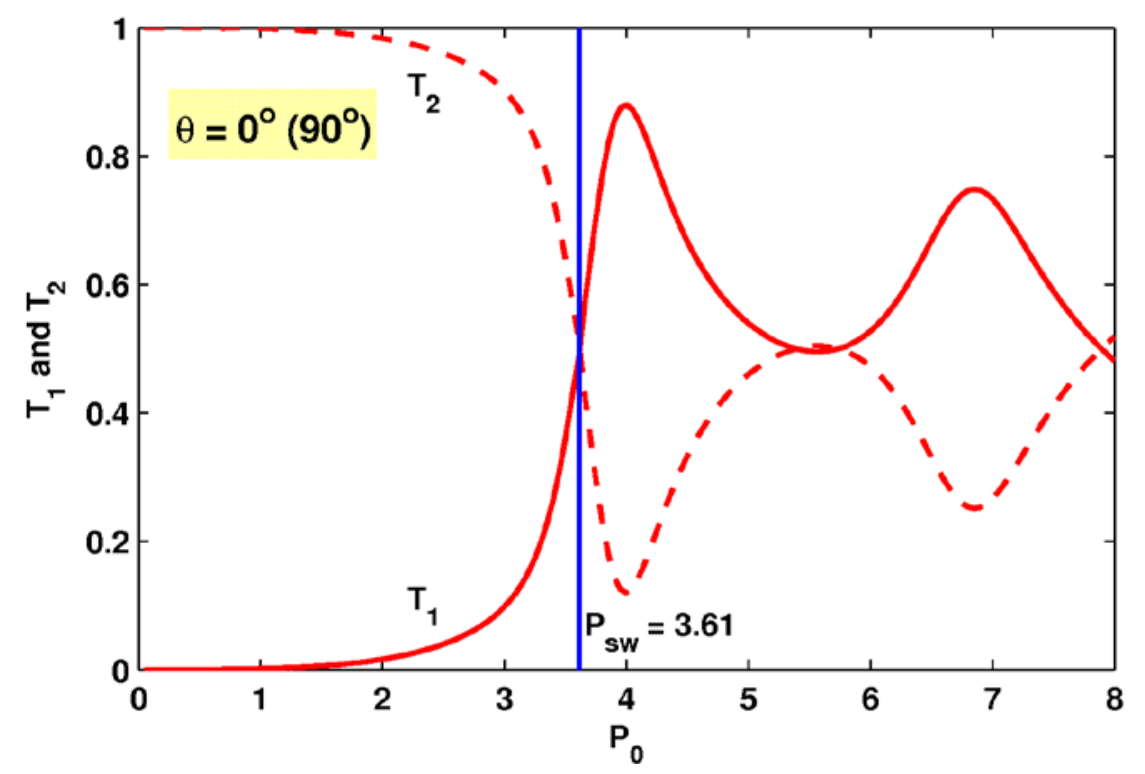

Fig. 1. 

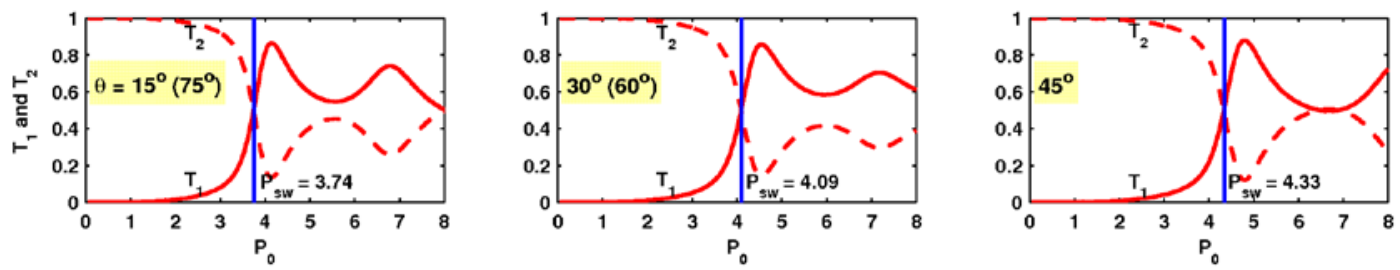

(a)
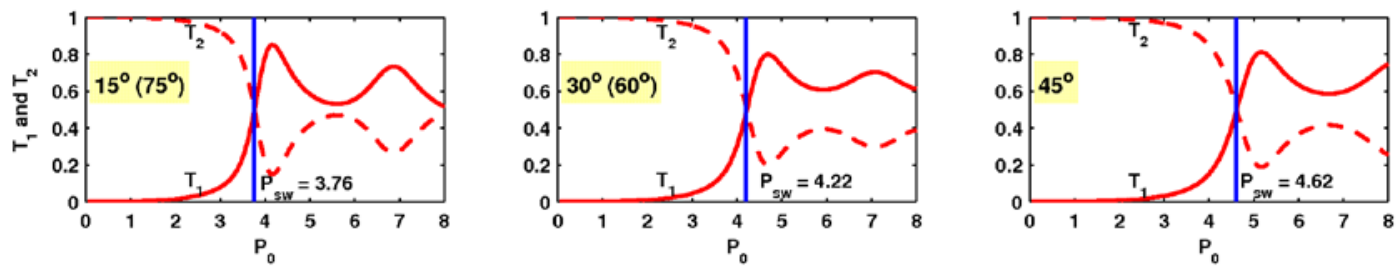

(b)
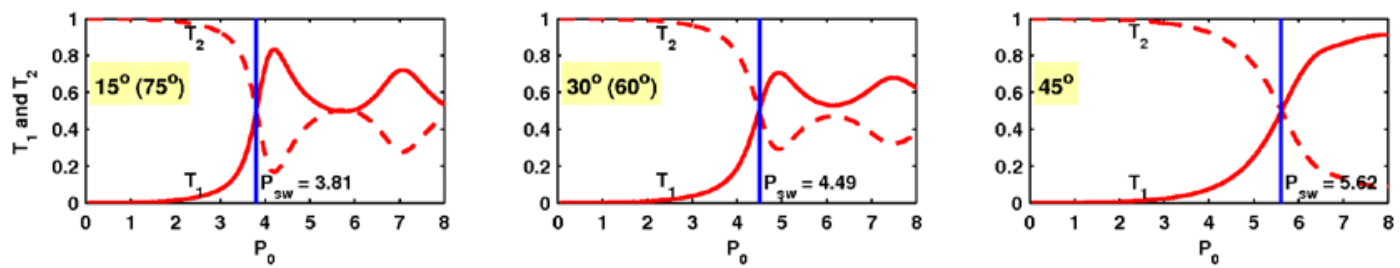

(c)
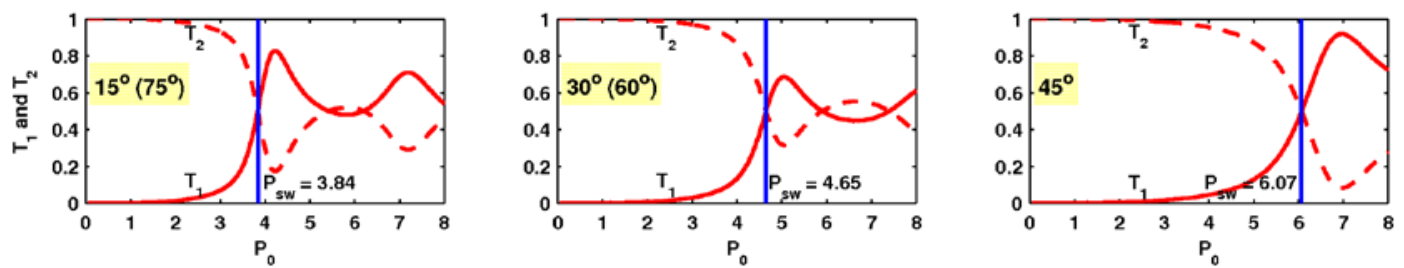

(d)
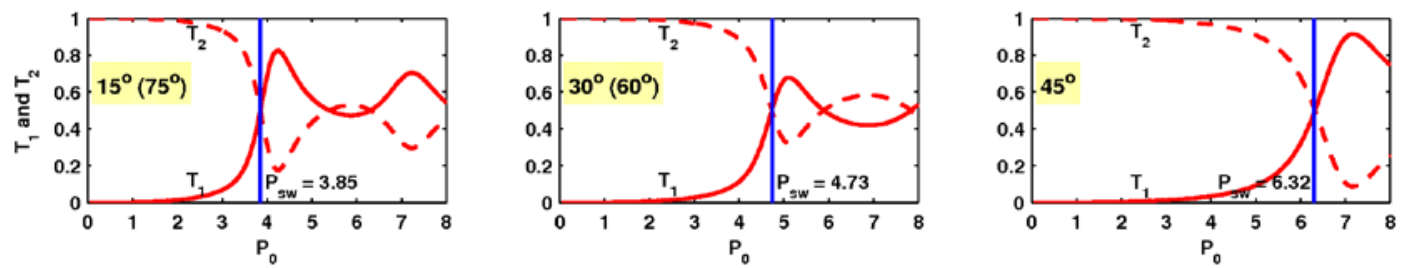

(e)
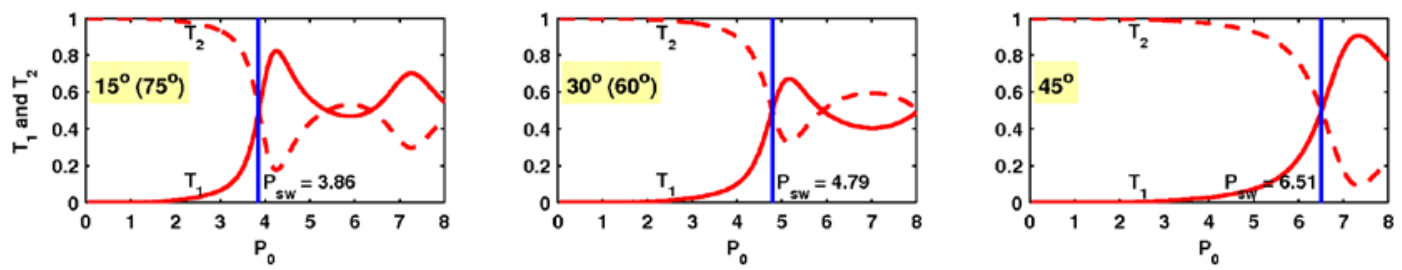

Fig. 2. 


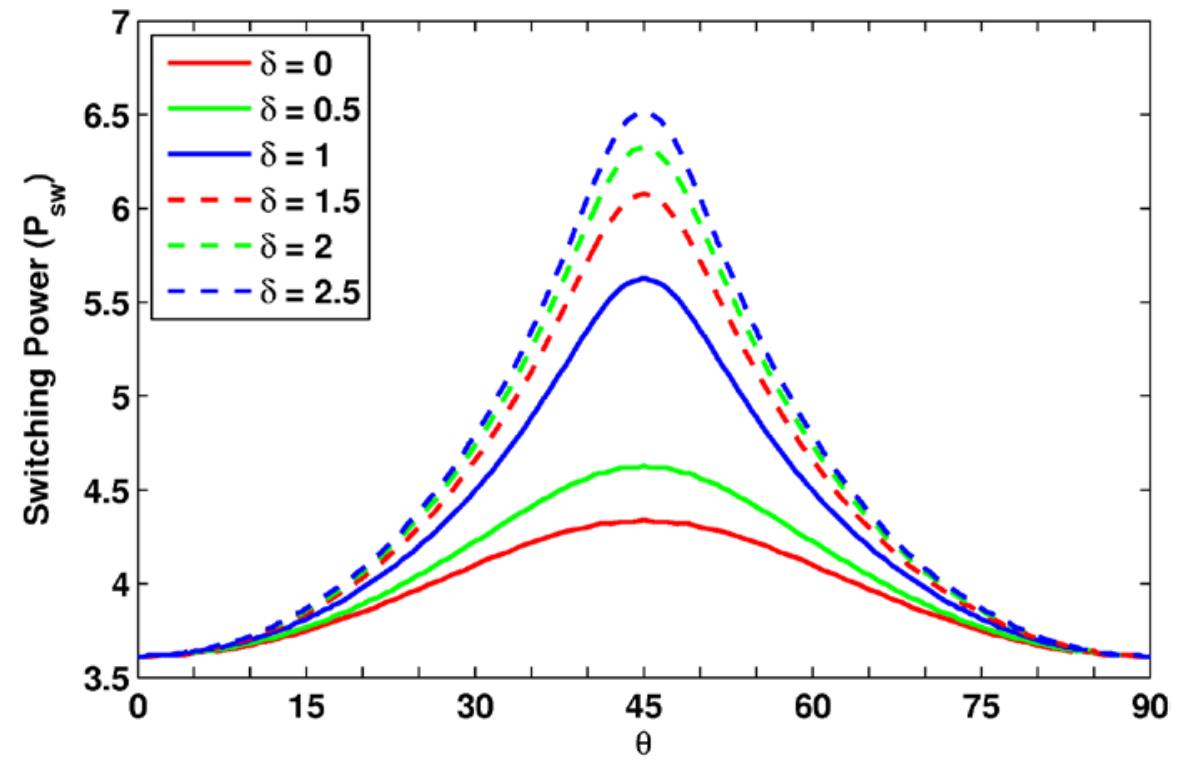

Fig. 3. 


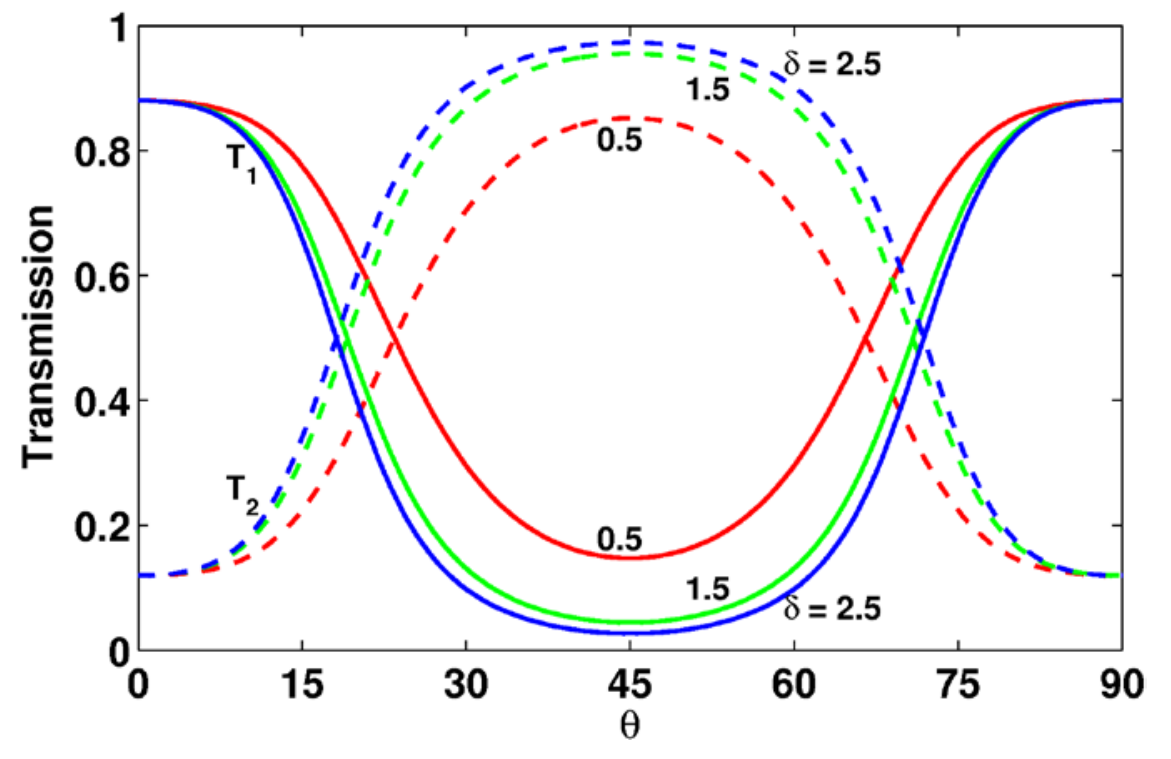

Fig. 4. 


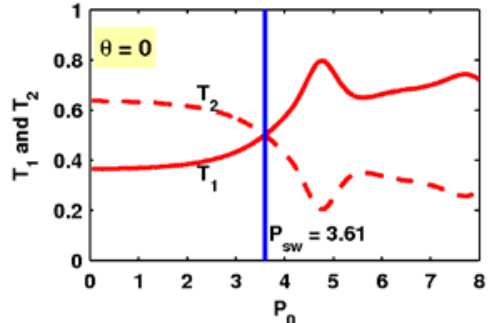

(a)

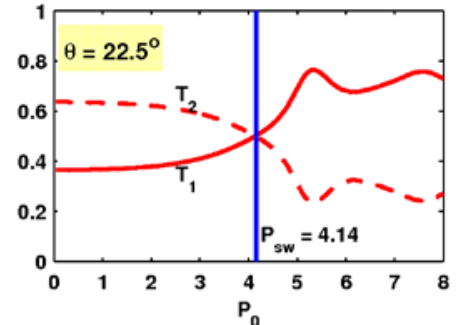

(b)

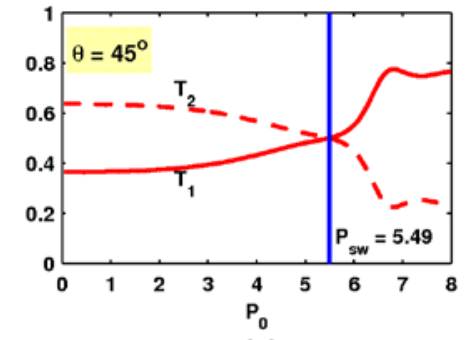

(c)

Fig. 5. 